\title{
ФИЗИЧЕСКАЯ ХИМИЯ
}

УДК 544.77.051

DOI 10.26456/vtchem2020.1.1

\section{К ВОПРОСУ О БАЗОВЫХ ПРИНЦИПАХ ПОСТРОЕНИЯ МЕЗОСКОПИЧЕСКОЙ МОДЕЛИ ЦИСТЕИН-СЕРЕБРЯНОГО PACTBOPA}

\author{
М.Д. Малышев ${ }^{1}$, П.О. Бабуркин ${ }^{1}$, Л.В. Жеренкова ${ }^{1}$ \\ П.М. Пахомов ${ }^{1}$, П.В. Комаров ${ }^{1,2}$
}

${ }^{1}$ ФГБОУ ВО «Тверской государственный университет» г. Тверь; ${ }^{2}$ ФГБУН «Институт элементоорганических соединений им. А. Н. Несмеянова РАН» г. Москва

\begin{abstract}
Исследована структура агрегатов меркаптида серебра (MC) в рамках крупномасштабного атомистического моделирования цистеинсеребряного раствора (ЦСР). Выполнен анализ строения, характеристик и потенциала взаимодействия супрамономеров в составе агрегатов МС. Эти данные являются основой для перехода на мезоскопический уровень моделирования ЦСР. В работе также рассмотрен вопрос о роли соли инициатора в нарушении электростатической стабилизации ЦСР.

Ключевые слова: гидрогель, атомистическая модель, мезоскопическая модель, молекулярная динамика.
\end{abstract}

Гидрогели (ГГ) и продукты на основе веществ в гелеобразном состояния широко распространены в повседневной жизни и применяются в различных технологических процессах [1-8]. С точки зрения природы межчастичного взаимодействия гели классифицируются на химические (формирование пространственной гель-сетки обусловлено образованием ковалентных связей между исходными реагентами) и физические (межмолекулярные связи являются обратимыми, т.е. их энергия сопоставима по порядку величины с энергией теплового движения $k_{B} T$, где $k_{B}$ - постоянная Больцмана, $T$ - абсолютная температура) [6].

К числу обратимых супрамолекулярных систем относится исследуемый нами водный раствор L-цистеина и нитрата серебра ЦСР. Уникальность ЦСР заключается в относительной простоте его получения, а также в способности к формированию биосовместимого физического супрамолекулярного ГГ при достаточно низком содержании дисперсной фазы ( 0.01\%) [9-11]. По сути, ЦСР представляет собой супраполимерную систему, способную менять и восстанавливать свою структуру при изменении внешнего воздействия - механических нагрузок и температуры. Эти свойства вместе с 
биосовместимостью и бактерицидным действием позволяют рассматривать ЦСР как перспективную основу для разработки биомедицинских препаратов наружного использования. Кроме этого, данная система является удобной для изучения закономерностей процессов самосборки в супрамолекулярных системах.

Многочисленные экспериментальные данные [9-12] по исследованию ЦСР свидетельствуют о том, что при сливании водных растворов L-цистеина и нитрата серебра происходит замещение атома водорода тиольной группы серебром, в результате чего формируются молекулы меркаптида серебра. Затем при «созревании» ЦСР происходит формирование заряженных кластеров МС с различными радиусами инерции (1-600 нм), что подтверждается результатами динамического рассеяния света (ДРС) и потенциометрии $[10,12]$. Важно отметить, что гелеобразное состояние на основе ЦСР получают после процесса созревания при добавлении различных солей, диссоциирующих с образованием как однозарядных, так и двухзарядных анионов, и катионов металлов. $\mathrm{C}$ помощью просвечивающей электронной микроскопии (ПЭМ) было показано, что гидрогели на основе ЦСР имеют сетчатую структуру (рис. 1.). При этом сами волокна гель-сетки состоят из отдельных хорошо различимых кластеров. Несмотря на большой объем данных по свойствам ЦСР и гелей на его основе, некоторые вопросы относительно понимания механизма гелеобразования в этой системе требуют отдельного изучения $[13,14]$. В частности, не совсем понятна роль соли инициатора гелеобразования в формировании супраполимерных цепочек.
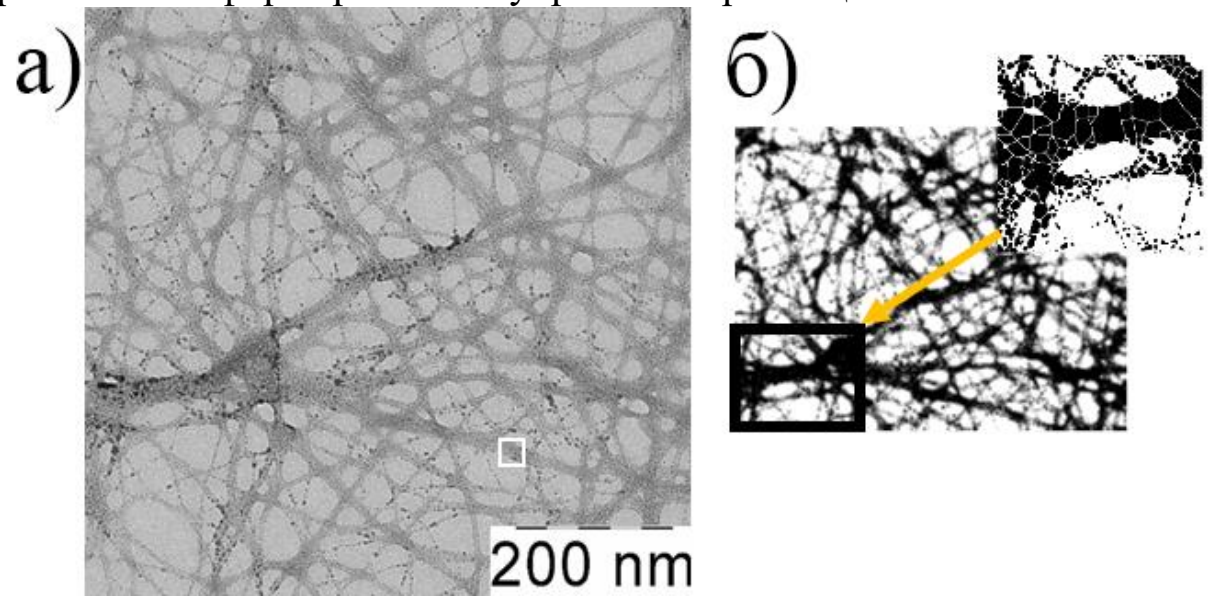

Рис. 1. а) Микрофотография образца гидрогеля на основе цистеина, $\mathrm{AgNO}_{3}$ и $\mathrm{Na}_{2} \mathrm{SO}_{4}\left(C_{\mathrm{Cys}}=3,0 \mathrm{MM}, C_{\mathrm{AgNO}}=3,75 \mathrm{mM}, C_{\mathrm{Na} 2 \mathrm{SO} 4}=0,2 \mathrm{мM}\right)$. Белый квадрат схематически показывает соотношение размеров ячейки моделирования с масштабами реальных агрегатов; б) обработка микрофотографии с использованием программы ImageJ [15] 
В работе [16] мы смоделировали стадию «созревания» ЦСР. Это позволило явно наблюдать формирование агрегата на основе молекул меркаптида серебра в ячейке моделирования $(215 \AA)^{3}$, размер которого сопоставим с масштабами реальных агрегатов (рис. 1). Однако реализация следующего шага - моделирования гелеобразования под влиянием солей инициаторов - представляет собой достаточно времяемкую задачу. Например, для моделирования ЦСР в течение 220 нс в отсутствие соли было затрачено 27000 процессорочасов. В данной работе мы задаемся целью сформулировать базовые принципы построения мезоскопической модели ЦСР на основе информации полученной в рамках метода молекулярной динамики [16] и выполнить ее параметризацию.

В качестве первого шага при построении мезоскопической модели ЦСР мы выполнили анализ структуры агрегата меркаптида серебра на основе данных продуктивной траектории атомистического моделирования, полученной в работе [16]. Для проведения анализа из ячейки моделирования были удалены все компоненты системы, не входящие в состав агрегатов МС. Затем было выполнено разбиение агрегатов на отдельные кластеры. При выполнении разбиения мы исходили из результатов работы [17], в которой исследовалась внутренняя структура кластеров, содержащих различное число молекул МC. Стабилизация исследованных кластеров происходит за счет образования молекулами МС тиол-серебряных олигомерных цепочек в результате формирования связей между атомами серы и серебра соседних молекул [17]. Длины образующихся связей лежат в диапазоне 2-3 А̊. Таким образом, алгоритм процедуры разбиения агрегата МС серебра был основан на поиске и объединении в один кластер всех молекул МC, между которыми можно сформировать связи Ag-S с длиной не больше $3 \AA$. На основе выполненного разбиения мы можем констатировать факт, что образующиеся в ходе МД расчетов агрегаты состоят из множества отдельных кластеров $\mathrm{MC}$ с различными радиусами инерции (рис. 2).

Все выделенные кластеры МС имеют одинаковую структуру, которую можно охарактеризовать как «ядро-оболочка» (рис. 3). Ядро кластеров преимущественно состоит из тиол-серебряных олигомерных цепочек, a поверхность (оболочка) содержит большое число функциональных групп $\mathrm{NH}_{3}{ }^{+}$и $\mathrm{C}(\mathrm{O}) \mathrm{O}^{-}$(рис. 3a). Этот результат хорошо согласуется с выводами работы [17]. На рис. 36 представлен фрагмент агрегата MC, в котором удалены части молекул $\mathrm{MC}$, образующие поверхность. На рисунке хорошо видно наличие свободного пространства между ядрами кластеров, что указывает на образование 
перекрестных водородных связей между $\mathrm{NH}_{3}{ }^{+}$и $\mathrm{C}(\mathrm{O}) \mathrm{O}^{-}$группами на поверхности.
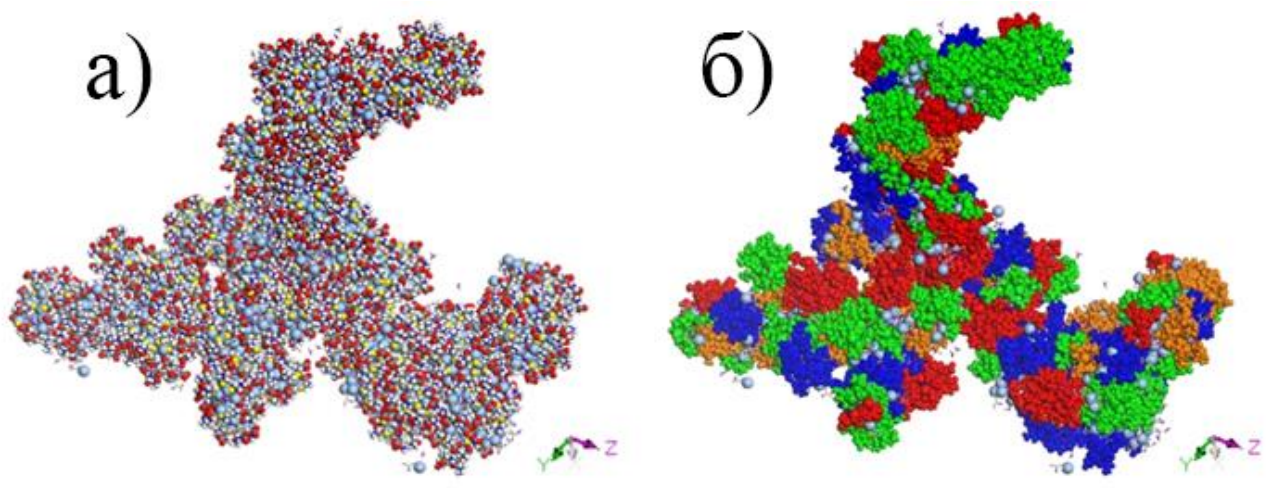

Рис. 2. Пример мгновенного снимка агрегата МС полученного в [16]: а) до разбиения на кластеры, б) после разбиения на кластеры

Исходя из этого можно сделать вывод, что выделенные кластеры МC можно расценивать как супрамономеры, из которых формируются крупномасштабные агрегаты.

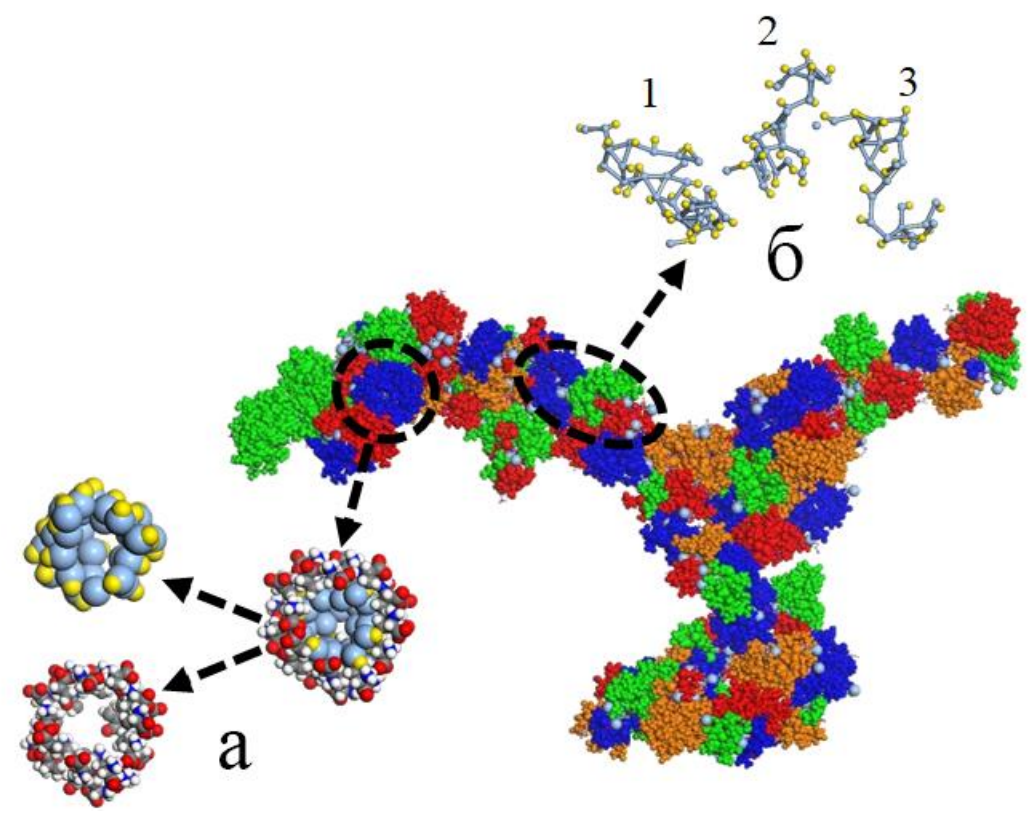

Рис. 3. Структурные особенности кластеров МС результирующего агрегата: а) кластер меркаптида серебра в разрезе; б) фрагмент цепного агрегата, состоящий из трех кластеров МС 
На следующем шаге была выполнена оценка величины заряда $Q / e$ (рис. 4 a) и радиуса инерции $R_{i}$ (рис. 46 ) кластеров в зависимости от числа молекул меркаптида серебра $N_{M C}$ в их составе. Как видно из рис. 4 , их заряд и радиус инерции $R_{i}$ стремятся к насыщению при увеличении $N_{M C}$. Средний $R_{i}$ равен $7.0 \pm 1.81 \AA$, а среднее значение заряда составляет $3 \pm 2.8$. Необходимо отметить, что возникновение положительного заряда обусловлено включением избыточных ионов $\mathrm{Ag}^{+}$раствора (образованных при диссоциации нитрата серебра) в состав кластеров МС.

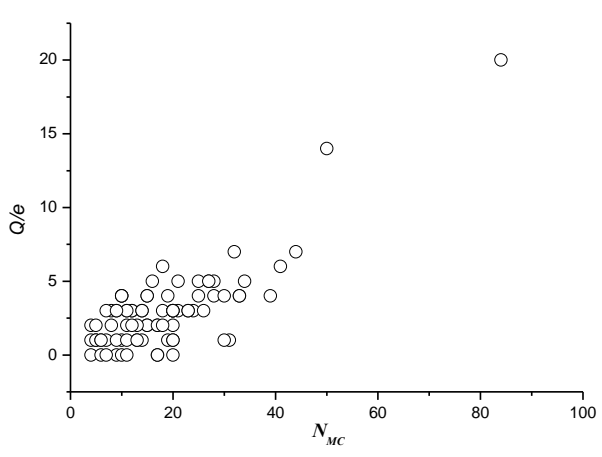

a)

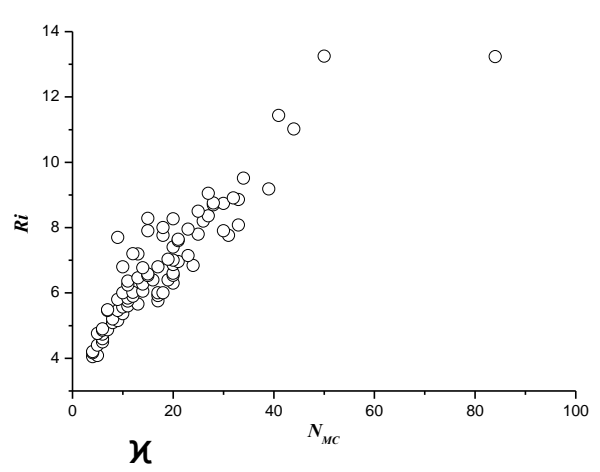

б)

Рис. 4. а) Заряд $(Q / e)$ кластеров и б) радиус инерции $\left(R_{i}\right)$ кластеров нормированные на число молекул меркаптида серебра $\left(N_{M C}\right)$ в их составе

Сформулируем общие принципы построения мезоскопической модели ЦСР. Мы будем полагать, что созревший ЦСР состоит из сферических частиц (супрамономеров) одинакового заряда $q=3 e$ и диаметра $\sigma=14 \AA$, в дальнейшем используемого как единица длины. Растворитель будем рассматривать как фоновую среду, свойства которой определяются концентрацией ионов, не входящих в состав кластеров. Предположение о том, что все ионы входят в состав среды, будем использовать в ходе первого этапа построения мезоскопической модели. Исходя из специфики строения кластеров МС будем полагать, что их можно рассматривать как “липкие” частицы. Таким образом взаимодействие между двумя кластерами меркаптида серебра с номерами $\alpha$ и $\beta$, разделенных расстоянием $r \equiv\left|\mathbf{r}_{\alpha}-\mathbf{r}_{\beta}\right|$, определяется суммой короткодействующего потенциала Леннарда-Джонса $\left(U^{(L /)}\right)$ и экранированного кулоновского потенциала $\left(U^{(q)}\right)$

$$
U_{\alpha \beta}(r)=U^{(L j)}(r)+U^{(q)}(r)
$$


Вестник ТвГУ. Серия "Химия". 2020. № 1 (39).

$$
U^{(L j)}(r)=4 \varepsilon\left[\left(\frac{\sigma}{r}\right)^{m}-\left(\frac{\sigma}{r}\right)^{n}\right] J U^{(q)}(r)=\frac{L_{B} q_{\alpha} q_{\beta}}{r} e^{-x r},
$$

где $r$ - расстояние между центрами кластеров; $\varepsilon$ - глубина потенциальной ямы; $\sigma$ - диаметр кластера; $\mathrm{q}_{i}-$ заряды кластеров; $L_{B}-$ длина Бьеррума; $x$-константа Дебая-Хюккеля. Длина Бьеррума задается как $L_{B}=e^{2} /\left(4 \pi \varepsilon_{0} \varepsilon \sigma k_{B} T\right)$, где $\sigma$ - диаметр кластера; $k_{B}$ постоянная Больцмана; $T$ - абсолютная температура; $e$ - элементарный заряд; $\varepsilon_{0}$ и $\varepsilon$ - диэлектрическая проницаемость вакуума и среды соответственно. Как уже было отмечено выше, присутствие соли инициатора гелеобразования мы учитываем как свойство среды. Для этого в случае использования потенциала (3) можно ввести параметр экранирования $x$ (обратный радиусу экранирования Дебая)

$$
x^{2}=4 \pi L_{B} \sum_{i} \frac{N(\mathrm{C}) * z_{i}^{2}}{(L / \sigma)^{3}}
$$

где $z_{i}$ - заряд противоиона; $L$ - длина ребра ячейки моделирования $N\left(C_{i}\right)$ - число противоионов $\left(\mathrm{NO}_{3}^{-}, \mathrm{Ag}^{+}, \mathrm{H}_{3} \mathrm{O}^{+}\right)$, не входящих в состав агрегата, рассчитанные исходя из концентраций реагентов, при которых образуется наиболее прочный гидрогель, $\mathrm{C}_{\mathrm{AqNO}}$ $=3.85 \mathrm{мМоль/л,} \mathrm{C}_{\text {суs }}=3 \mathrm{мМоль/л} \mathrm{[14].}$

Восстановление короткодействующего потенциала ЛеннардаДжонса было выполнено в рамках молекулярно-механических расчетов с использованием валентно-силового поля PCFF [18]. Для определения констант LJ были построены три пары кластеров, состоящих из разного числа молекул МС (рис. 5.):

$$
\mathrm{N}_{\mathrm{MC}}=15\left(\sigma_{1}=18 \AA\right) ; \mathrm{N}_{\mathrm{MC}}=23\left(\sigma_{2}=20 \AA\right) ; \mathrm{N}_{\mathrm{MC}}=36\left(\sigma_{3}=24 \AA\right) \text {. }
$$

Для каждой пары кластеров было построено пять конфигураций отличающихся взаимной ориентацией кластеров, которая задавалась случайным образом. Энергия взаимодействия кластеров каждой конфигурации вычислялась как функция расстояния $r$ между их центрами

$$
\Delta E i\left(r, N_{\mathrm{MC}}\right)=\left|\operatorname{Ei}\left(r, N_{\mathrm{MC}}\right)-\operatorname{Ei}\left(\infty, N_{\mathrm{MC}}\right)\right|,
$$

где $\Delta E i\left(r, N_{\mathrm{MC}}\right)$ - энергия взаимодействия кластеров, состоящих из $N_{\mathrm{MC}}$ молекул $\mathrm{MC}, E\left(r, N_{\mathrm{MC}}\right)$ - полная энергия системы, $\operatorname{Ei}\left(\infty, N_{\mathrm{MC}}\right)-$ энергия кластеров, когда $r=100 \AA$, $i$ - номер конфигурации. Результирующая энергия взаимодействия кластеров вычислялась посредством усреднения по всем построенным конфигурациям. Установлено, что энергия взаимодействия кластеров хорошо аппроксимируется потенциалом $U^{(L /)}$, когда ${ }^{\varepsilon} / k_{\mathrm{B}} T=48.2, m=22$, а $n=$ 11. При этом глубина потенциальной ямы $\varepsilon$ изменяется 
пропорционально $\sigma^{2}$ (рис. 6а), что указывает на то, что сила взаимодействия кластеров МС в однородной среде пропорциональна площади контакта поверхностей кластеров $S_{\mathrm{c}}(\sigma)$. Таким образом,

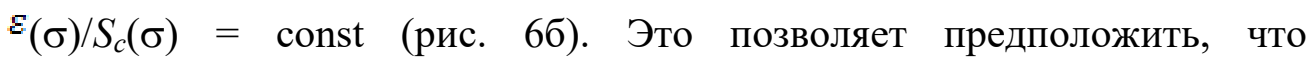
короткодействующее взаимодействие кластеров МC определяется только их размером.
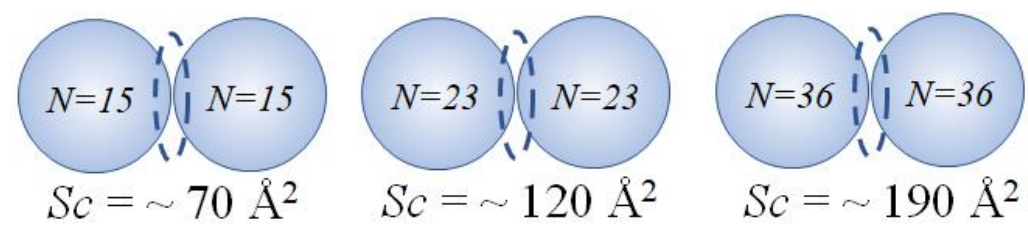

Рис. 5. Схематическая иллюстрация использованных кластеров при параметризации констант потенциала Леннарда-Джонса. Sc - площадь контакта
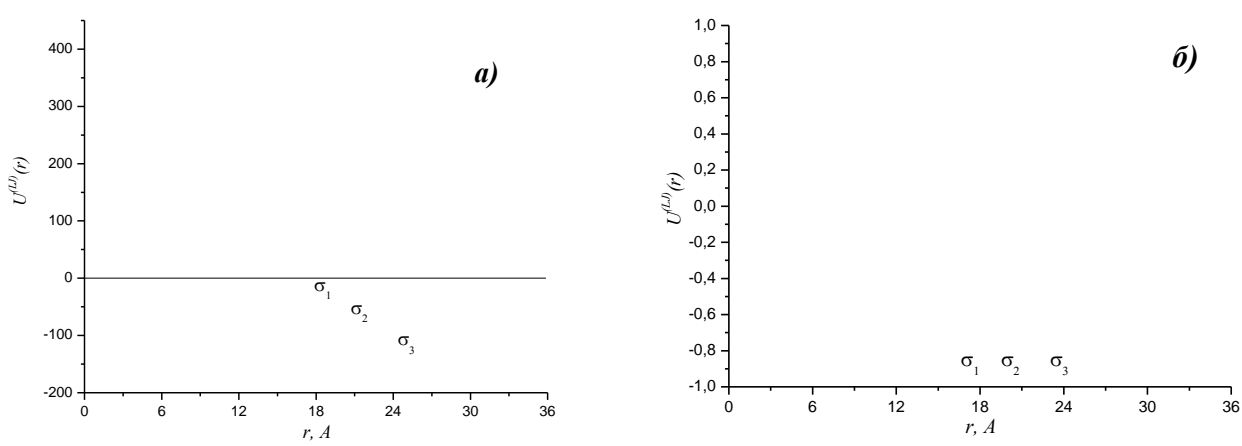

Рис. 6. а) Потенциалы Леннард-Джонса, восстановленные для пар взаимодействующих кластеров $\mathrm{MC}: \sigma_{1}=18 \AA\left(\mathrm{S}_{\mathrm{c}}=70 \AA^{2}\right), \sigma_{2}=20 \AA\left(\mathrm{S}_{\mathrm{c}}=\right.$ $\left.120 \AA^{2}\right), \sigma_{3}=24 \AA\left(\mathrm{S}_{\mathrm{c}}=190 \AA^{2}\right)$, б) те же потенциалы, нормированные на площадь поверхности контакта кластеров $\left(\mathrm{S}_{\mathrm{c}}\right)$

На рис. 7 показано поведение потенциальной энергии взаимодействия кластеров МС (в единицах $k_{\mathrm{B}} T$ ) в зависимости от расстояния между ними при различных концентрациях соли $\left(a_{1}-\right.$ 0 мМоль/л, $a_{2}-0.5$ мМоль/л, $a_{3}-10$ мМоль/л). Как видно из рисунка, в области малых расстояний $\left(1 \leq r^{\sigma} \leq 1.4\right)$ потенциальная энергия имеет вид глубокой потенциальной ямы, обусловленной притяжением кластеров вследствие взаимодействия функциональных групп на их поверхности. В области средних расстояний $\left(1.4 \leq r^{\sigma} \leq 3\right)$ хорошо виден потенциальный барьер, возникающий в результате наличия у кластеров положительных зарядов. Когда в системе нет соли инициатора гелеобразования, величина потенциального барьера составляет $\sim k_{\mathrm{B}} T$, что сопоставимо с масштабом энергии теплового 
движения в системе. Очевидно, что для выделенных нами супрамономеров такой энергетический барьер не должен являться препятствием для формирования вторичных надмолекулярных структур. При этом, благодаря кулоновскому отталкиванию на масштабах $r / \sigma \sim 2$, энергетически выгодным является формирование вытянутых морфологий, как это и наблюдается в случае структуры, показанной на рис. 2. Отметим, что при больших концентрациях соли, когда потенциальный барьер полностью исчезает (см. рис. 7), супрамономеры могут слипаться в агрегаты произвольной формы и размера, что отвечает экспериментально наблюдаемому режиму коагуляции ЦСР.

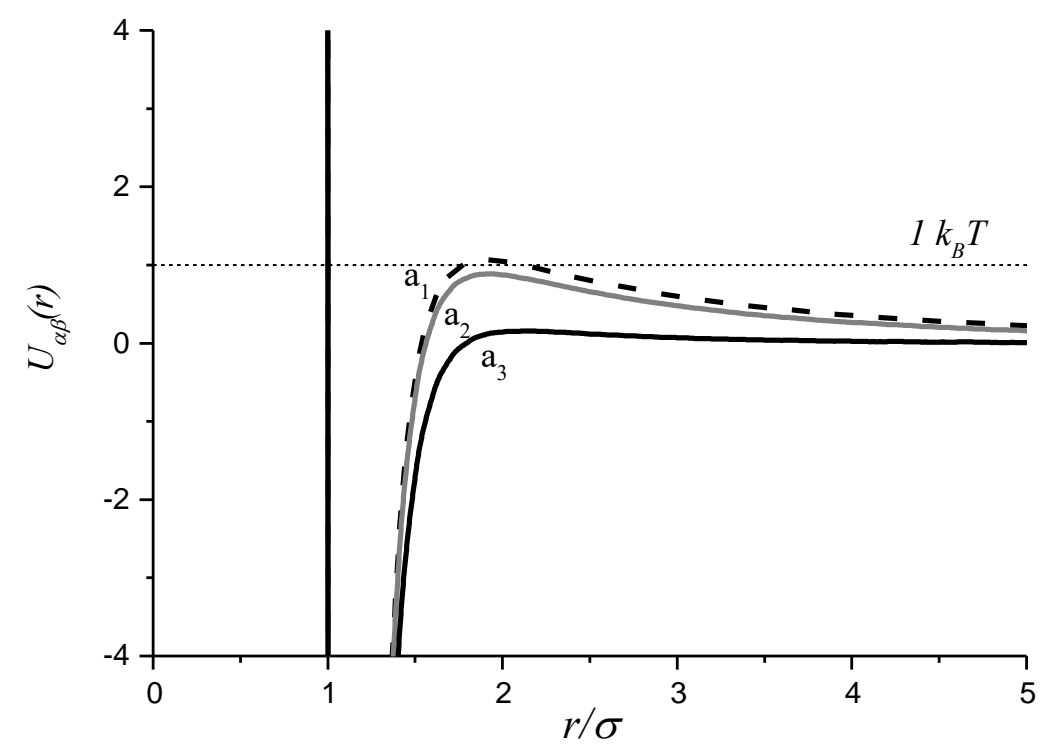

Рис. 7. Парный потенциал взаимодействия кластеров МС (в единицах $k_{\mathrm{B}} T$, ) при различных концентрациях соли: $a_{1}-0$ мМоль/л $(\varkappa=0.24), a_{2}-$ 0.5 мМоль/л $(\varkappa=0.30), a_{3}-10$ мМоль/л $(\varkappa=0.83)$

Таким образом, в данной работе нам удалось качественно проиллюстрировать причины роста вытянутых агрегатов в процессе созревания ЦСР. Исходя из выполненного анализа, можно заключить, что гелеобразование в ЦСР является многоуровневым процессом, в котором можно выделить три последовательных этапа. Сначала в ЦСР образуются положительно заряженные кластеры меркаптида серебра с диаметром $\sim 14 \AA$ ( этаn), которые впоследствии (в ходе созревания ЦСР) формируют крупномасштабные агрегаты (II эman) за счет связывания кластеров МС посредством водородных связей между функциональными группами на их поверхности. Размер образующихся вторичных агрегатов контролируется их зарядом. Непосредственно 
гелеобразование происходит за счет объединения образовавшихся агрегатов в волокна гель-сетки при добавлении соли инициатора (III этаn). Образующиеся волокна гель-сетки можно рассматривать как третичные агрегаты. Их нитеобразная форма должна быть обусловлена большим зарядом вторичных агрегатов. Оценка дебаевского радиуса при наличии и отсутствии соли инициатора $[9,10]$ дает значения $\sim 46.7 \AA$ и $\sim 48 \AA$ соответственно. Это позволяет заключить, что гелеобразование в ЦСР происходит не за счет снижения электростатической стабилизации вторичных агрегатов МC, а по другой причине.

Мы полагаем, что разработанные элементы мезоскопической модели позволят нам выполнить изучение поведения ЦСР на больших пространственных масштабах. Главным вопросом следующего этапа моделирования будет выявление механизма нарушения электростатической стабилизации вторичных супрамолекулярных агрегатов при введении соли инициатора.

\section{Благодарности}

Работа выполнена с использованием оборудования Центра коллективного пользования сверхвысокопроизводительными вычислительными ресурсами МГУ имени М.В. Ломоносова [19], при поддержке Министерства науки и высшего образования Российской Федерации и финансовой поддержке Российского фонда фундаментальных исследований (проект № 18-33-00146 мол_а).

\section{Список литературы}

1. Brunsveld L., Folmer B. J. B., Meijer E. W., Sijbesma R. P. // Chem. Rev. 2001. V. 101. № 12. P. 4071-4097.

2. Estroff L., Hamilton A.D. // Chem. Rev. 2004. V. 104. № 3. P. 1201-1218.

3. Sangeetha N.M., Maitra U. // Chem. Soc. Rev. 2005. V. 34. № 10. P. 821-836.

4. Peppas N.A., Hilt J.Z., Khademhosseini A., Langer R. // Advanced Materials. 2006. V. 18. № 11. P. 1345-1360.

5. Serpe M.J., Craig S.L. // Langmuir. 2007. V. 23. № 4. P. 1626-1634.

6. Maity G.C. // Journal of Physical Sciences. 2007. V. 11. № 1. P. 156-171.

7. Loos M., Feringa B.L., Esch J.H. // Eur. J. Org. Chem. 2005. V. 2005. № 17. P. 3615-3631.

8. Liu K., Kang Y., Wang Z., Zhang X. // Adv. Mater. 2013. V. 25. № 39. P. 55305548.

9. Пахомов П.М., Овчинников М.М., Хижняк С.Д., Лавриенко М.В., Nierling W., Lechner M.D. // Коллоидный журнал. 2004. T. 66, № 1. C. 75-79.

10. Пахомов П.М., Овчинников М.М., Хижняк С.Д., Рощина О.А., Комаров П.В. // Высокомол. соединения. Сер. А. 2011. Т.53. № 9. С. 1574-1581.

11. Баранова О.А., Кузьмин Н.И., Самсонова Т.И., Ребецкая И.С., Петрова О.П., Пахомов П.М., Хижняк С.Д., Комаров П.В., Овчинников М.М. // Химические волокна. 2011. № 1. С. 74-86. 
Вестник ТвГУ. Серия "Химия". 2020. № 1 (39).

12. Алексеев В.Г., Семенов А.Н., Пахомов П.М. // Журнал неорганической химии. 2012. Т. 57. № 7. С. 1115-1118.

13. Комаров П.В. // Использование многомасштабного моделирования для изучения свойств нанодисперсных полимерных систем: Монография Тверь: Твер. гос. ун-т., 2014. С. 256.

14. Пахомов П.М., Хижняк С.Д., Овчинников М.М., Комаров П.В. // Супрамолекулярные гели: Монография - Тверь: Твер. гос. ун-т., 2011. С. 272.

15. ImageJ // https://imagej.net/W

16. Малышев М.Д., Бабуркин П.О., Адамян А.Н., Хижняк С.Д., Пахомов П.М. // Журнал структурной химии. 2019. №8. С. 103-113.

17. Малышев М.Д., Комаров П.В., Хижняк С.Д., Пахомов П.М. // Вестник ТвГУ. Серия «Химия». 2017. №1. С. 103-113.

18. H. Sun. // Macromolecules. 1995. V. 28. P. 701-712.

19. Воеводин Вл.В., Жуматий С.А., Соболев С.И., Антонов А.С., Брызгалов П.А., Никитенко Д.А., Стефанов К.С., Воеводин Вад.В. // Открытые системы. 2012. № 7. С. 36-39.

\section{Об авторах:}

МАЛЫШЕВ Максим Дмитриевич - аспирант кафедры физической химии, ФГБОУ ВО «Тверской государственный университет», e-mail: bggf@bk.ru

БАБУРКИН Павел Олегович - аспирант кафедры физической химии, ФГБОУ ВО «Тверской государственный университет», e-mail: guppi_oc@mail.ru.

ЖЕРЕНКОВА Любовь Витальевна - кандидат физико-математических наук, доцент кафедры общей физики, ФГБОУ ВО «Тверской государственный университет»,, е-таil:введите е-mail

ПАХОМОВ Павел Михайлович - доктор химических наук, профессор, зав. кафедрой физической химии, ФГБОУ ВО «Тверской государственный университет», e-mail: pavel.pakhomov@mail.ru

КОМАРОВ Павел Вячеславович - доктор физико-математических наук, профессор кафедры общей физики, ФГБОУ ВО «Тверской государственный университет»; ведущий научный сотрудник лаборатории физической химии полимеров ФГБУН «Институт элементоорганических соединений им. А. Н. Несмеянова РАН», e-mail: pv_komarov@mail.ru 


\section{TO THE QUESTION OF BASIC PRINCIPLES OF CONSTRUCTION OF THE MESOSCOPIC MODEL OF THE CISTEINE-SILVER SOLUTION}

M. D. Malyshev ${ }^{1}$, P. O. Baburkin ${ }^{1}$, L. V. Zherenkova ${ }^{1}$, P.M. Pakhomov ${ }^{1}$, P. V. Komarov ${ }^{1,2}$

${ }^{1}$ Tver State University

${ }^{2}$ A.N. Nesmeyanov Institute of Organoelement Compounds of Russian Academy of Sciences

The analysis of the structure of silver mercaptide (SM) aggregates obtained in the framework of large-scale atomistic modeling of L-cysteine and silver nitrate solution (CSS) was performed. Information was obtained on the structure, characteristics and interaction of supramonomers forming SM aggregates. These data can serve as the basis for the transition to the mesoscopic level of CSS simulations The paper also examined the role of the initiator salt in disrupting the electrostatic stabilization of the CSS.

Keywords: hydrogel, atomistic model, mesoscopic model, molecular dynamics. 